\title{
Existence and uniqueness of solution for class of fractional order differential equations on an unbounded domain
}

\author{
Azizollah Babakhani
}

Correspondence: babakhani@nit.ac

Department of Mathematics, Faculty of Basic Science, Babol University of Technology, Babol 47148-71167, Iran

\begin{abstract}
In this article, we established the existence and uniqueness of the solution for a generalized class of fractional order differential equations involving the RiemannLiouville differential operator on unbounded domain $[0,+\infty)$. The contraction principle has been used to obtain the results in this article.
\end{abstract}

Keywords: fractional differential equations, gamma function, Voltra integral equation

\section{Introduction}

Differential equations of fractional order have recently proved to be valuable tools in the modeling of many phenomena in various fields of science and engineering. Indeed, we can find numerous applications in viscoelasticity, electrochemistry, control, porous media, electromagnetism, etc[1-5]. There has been a significant development in the study of fractional differential equations and inclusions in recent years; see the monographs of Kilbas et al. [6], Lakshmikantham et al. [7], Podlubny [4], and the survey by Agarwal et al. [8]. For some recent contributions on fractional differential equations, see [9-28] and the references therein. Very recently in $[10,11,21,22]$ the authors and other researchers studied the existence and uniqueness of solutions of some classes of fractional differential equations with delay. For more details on the geometric and physical interpretation for fractional derivatives of both the Caputo types see $[5,23]$. Baleanu and Mustafa [16] have considered

$$
\left\{\begin{array}{l}
D^{\alpha}(x(t)-x(0))=f(t, x(t)), \quad t>0, \\
x(0)=x_{0}
\end{array}\right.
$$

where $D^{\alpha}$ is the standard Riemann-Liouville fractional derivatives and $f: \mathbb{R}^{\geq 0} \times \mathbb{R} \rightarrow$ $\mathbb{R}$ is a given continuous function. They have shown that the initial value problem (1) has unique solution in $\mathbb{R}^{\geq 0}=[0, \infty)$, if $|f(t, x)-f(t, y)| \leq F(t)|x-y|$ for all $t \geq 0, x, y \in$ $\mathbb{R}$, where $F: \mathbb{R}^{\geq 0} \rightarrow \mathbb{R}^{\geq 0}$ is a continuous function.

In this article, we consider the following initial value problem:

$$
\left\{\begin{array}{l}
\mathcal{L}(D)(x(t)-x(0))=f(t, x(t)), \quad t>0, \\
x(0)=x_{0},\left.I^{1-\alpha} x(t)\right|_{t=0}=0,\left.I^{1-\beta} x(t)\right|_{t=0}=0,
\end{array}\right.
$$


where $\mathcal{L}(D)=D^{\alpha}-\gamma t^{n} D^{\beta}, n$ is nonnegative integer, $\gamma \in \mathbb{R}, 0<\beta \leq \alpha<1$ and $D^{\alpha}$ and $D^{\beta}$ are the standard Riemann-Liouville fractional derivatives. Here $f: \mathbb{R}^{\geq 0} \times \mathbb{R} \rightarrow \mathbb{R}$ is a given nonlinear continuous function and $\mathbb{R}^{\geq 0}$ has its usual meaning of nonnegative semi-axis. We prove the solution for Equation (2) is exist and under some conditions on $f(t, x)$ and on the range of function $F$, this solution is unique. Note that, Equation (1) is a particular case of Equation (2), that is if $\gamma=0$ then Equation (2) reduces to Equation (1).

The article is organized as follows. In Section 2, we give basic definitions of Riemann-Liouville fractional calculus. Existence and uniqueness solution have been derived in Section 3 and also an example is given to illustrate our results.

\section{Basic definitions and preliminaries}

Definitions of Riemann-Liouville fractional derivative/integral and some of their properties are given below which could be found in $[4,19,20]$.

Definition 2.1 The left sided Riemann-Liouville fractional integral of a function $x \mathrm{~L}$ $L^{1}([a, b], \mathbb{R})$ of order $\alpha>0$ is defined as

$$
I_{a^{+}}^{\alpha} x(t)=\frac{1}{\Gamma(\alpha)} \int_{a}^{t} \frac{x(s)}{(t-s)^{1-\alpha}} d s, \quad t>a,
$$

where $\Gamma(\alpha)=\int_{0}^{+\infty} e^{-t} t^{\alpha-1} d t$.

The fractional integral of $x(t)=(t-a)^{\beta}, a \geq 0, \beta>-1$ is given as

$$
I_{a^{+}}^{\alpha} x(t)=\frac{\Gamma(\beta+1)}{\Gamma(\beta+\alpha+1)}(t-a)^{\beta+\alpha} .
$$

Definition 2.2 The left sided Riemann-Liouville fractional derivative of a function $x$ defined as

$$
D_{a^{+}}^{\alpha} x(t)=\frac{d}{d t}\left\{I_{a^{+}}^{1-\alpha} x(t)\right\}, \quad \alpha \in(0,1) .
$$

We denote $D_{a^{+}}^{\alpha} x(t)$ by $D_{a}^{\alpha} x(t)$ and $I_{a^{+}}^{\alpha} x(t)$ by $I_{a}^{\alpha} x(t)$. Also $D^{\alpha} x(t)$ and $I^{\alpha} x(t)$ refer to $D_{0^{+}}^{\alpha} x(t)$ and $I_{0+}^{\alpha} x(t)$, respectively.

Proposition 2.3 [4, pp. 71-72] Suppose that $x(t)$ is continuous function on $[0, \infty)$ then $D_{0}^{\beta} x(t)$ is integrable,

$$
\begin{aligned}
& I^{\alpha}\left\{D^{\beta} x(t)\right\}=I^{\alpha-\beta} x(t)-\left[I^{1^{-\beta}} x(t)\right]_{t=0} \frac{t^{\alpha-1}}{\Gamma(\alpha)}, \quad 0<\beta<1, \quad \beta \leq \alpha, \\
& D^{\alpha}\left\{I^{\beta} x(t)\right\}=I^{\alpha-\beta} x(t) .
\end{aligned}
$$

Further, If $\left.I^{1-\beta} x(t)\right|_{t=0}=0$ then Equation (3) reduces to

$$
I_{0}^{\alpha}\left(D_{0}^{\beta} x(t)\right)=I_{0}^{\alpha-\beta} x(t), \quad 0<\beta<1, \beta \leq \alpha .
$$

Proposition 2.4 Let $\times$ be continuous on $[0,+\infty)$ and $0<\beta<1, \beta \leq \alpha$. If $\left.I^{1-\beta} x(t)\right|_{t=0}$ $=0$ then

(i) $I^{\alpha}\left(t^{n} x(t)\right)=\sum_{k=0}^{n}\left(\begin{array}{c}-\alpha \\ k\end{array}\right)\left[\frac{d^{k} t^{n}}{d t^{k}}\right]\left[I^{\alpha+k} x(t)\right]=\sum_{k=0}^{n}\left(\begin{array}{c}-\alpha \\ k\end{array}\right) \frac{n ! t^{n-k}}{(n-k) !} I^{\alpha+k} x(t)$, 
(ii) $I^{\alpha}\left(t^{n} D^{\beta} x(t)\right)=\sum_{k=0}^{n}\left(\begin{array}{c}-\alpha \\ k\end{array}\right) \frac{n ! t^{n-k}}{(n-k) !} I^{\alpha-\beta+k} x(t)$,

where $n$ is a nonnegative integer and,

$$
\left(\begin{array}{c}
-\alpha \\
k
\end{array}\right)=(-1)^{k} \frac{\alpha(\alpha+1) \cdots(\alpha+k-1)}{k !}=(-1)^{k} \frac{\Gamma(\alpha+k)}{k ! \Gamma(\alpha)}
$$

Proof. (i) can be found in [19, p. 53] and (ii) is an immediate consequence of Equation (3) and (i) with together $\left.I^{1-\beta} x(t)\right|_{t=0}=0$.

\section{Existence and uniqueness}

In this section, our objective is to find solutions to the initial value problem (2) with some conditions on $f(t, x)$ and on the range of function $F$ so that this solution is unique on unbounded domain $[0, \infty)$. First in the following lemma, we prove that the solution of Equation (2) is equivalent to the solution of an integral equation using Proposition 2.4.

Lemma 3.1 The solution of the initial value problem (2) is equivalent to the Voltra integral equation

$$
\begin{aligned}
x(t) & =x(0)+\gamma \sum_{k=0}^{n}\left(\begin{array}{l}
-\alpha \\
k
\end{array}\right) \frac{n ! t^{n-k}}{(n-k) !} I^{\alpha-\beta+k}[x(t)-x(0)]+I^{\alpha} f(t, x(t)), \quad t>0 \\
& =x(0)+\gamma \sum_{k=0}^{n} \mu(k, n, \alpha) t^{n-k} I^{\alpha-\beta+k}[x(t)-x(0)]+I^{\alpha} f(t, x(t)),
\end{aligned}
$$

where,

$$
\mu(k, n, \alpha):=\left(\begin{array}{c}
-\alpha \\
k
\end{array}\right) \frac{n !}{(n-k) !}, \quad k=0,1,2, \ldots, n .
$$

Proof: Suppose the Equation (2) is given, then $I^{\alpha}\left\{\left(D^{\alpha}-\gamma t^{n} D^{\beta}\right)[x(t)-x(0)]\right\}=I^{\alpha} f(x, x(t))$. With assumptions $\left.I^{1-\alpha} x(t)\right|_{t=0}=0$ and $\left.I^{1-\beta} x(t)\right|_{t=0}=0$, Equation (3) gives $I^{\alpha}\left\{D^{\alpha}[x(t)-x\right.$ $(0)]\}=x(t)-x(0)$ and using Proposition 2.4 (ii) the integral equation (4) is obtained (notice that $\left.I^{\alpha} x(0)\right|_{t=0}=\left.\frac{x(0)}{\Gamma(1+\alpha)} t^{\alpha}\right|_{t=0}=0$ ). Conversely, assume that the integral equation (6) is given hence

$$
\begin{aligned}
& \gamma \sum_{k=0}^{n}\left(\begin{array}{c}
-\alpha \\
k
\end{array}\right) \frac{n ! t^{n-k}}{(n-k) !} I^{\alpha-\beta+k}[x(t)-x(0)]+I^{\alpha} f(t, x(t)) \\
& =\gamma \sum_{k=0}^{n}\left(\begin{array}{c}
-\alpha \\
k
\end{array}\right)\left[D^{k} t^{n}\right]\left[I^{\alpha+k} D^{\beta}[x(t)-x(0)]+I^{1-\beta}[x(t)-x(0)]_{t=0}\right]+I^{\alpha} f(t, x(t)), \\
& =\gamma I^{\alpha}\left(t^{n} D^{\beta}[x(t)-x(0)]\right)+I^{\alpha} f(t, x(t)) \\
& =I^{\alpha}\left\{\gamma t^{n} D^{\beta}[x(t)-x(0)]+f(t, x(t)\}=x(t)-x(0)=I^{\alpha}\left\{D^{\alpha}[x(t)-x(0)]\right\} .\right.
\end{aligned}
$$

Notice that by using Equation (3) we have

$$
I^{\alpha}\left\{D^{\alpha}[x(t)-x(0)]\right\}=x(t)-x(0)-I^{1-\alpha}\{x(t)-x(0)\}_{t=0} .
$$

Hence the initial condition of $\left.I_{0}^{1-\alpha} x(t)\right|_{t=0}=0$ yields

$$
I^{\alpha}\left\{D^{\alpha}[x(t)-x(0)]\right\}=x(t)-x(0) .
$$


Thus $D^{\alpha}\left\{I^{\alpha}\left[\gamma t^{n} D^{\beta}[x(t)-x(0)]+f(x, x(t))\right]\right\}=D^{\alpha}\left\{I^{\alpha}\left(D^{\alpha}[x(t)-x(0)]\right)\right\}$. Therefore $\gamma t^{n} D^{\beta}[x(t)-x$ $(0)]+f(x, x(t))=D^{\alpha}[x(t)-x(0)]$ and we obtain the Equation (2). This proves that $x(t)$ is a solution of initial value problem (2) and the proof is complete. In the following theorem, we try to prove that the integral equation (6) under some conditions has unique solution in $[0, \infty)$ so that this unique solution is the unique solution of Equation (2).

Theorem 3.2 Assume that there exists the continuous function $F: \mathbb{R}^{\geq 0} \rightarrow[1,+\infty)$ such that $|f(t, x)-f(t, y)| \leq F(t)|x-y|$ for all $t \geq 0, x, y \in \mathbb{R}$ (notice that $F(t) \geq 1$ for each $t$ $\geq 0$ ). Then the integral equation (5) has unique solution defined in $\mathbb{R}^{\geq 0}$.

Proof. Introduce the continuous functions

$$
\begin{aligned}
h(t) & =1+|x(0)|+\gamma \sum_{k=0}^{n} \mu(k, n, \alpha) t^{n-k} I^{\alpha-\beta+k}[x(0)-x(0)]+I^{\alpha} f(t, x(0)) \\
& =1+|x(0)|+\frac{1}{\Gamma(\alpha)} \int_{0}^{t} \frac{|f(s, x(0))|}{(t-s)^{1-\alpha}} d s,
\end{aligned}
$$

and

$$
\left.H_{\lambda}(t)=h(t) \exp \left((n+1) t+\frac{\lambda}{q} \int_{0}^{t}[h(s) F(s)]^{q} d s\right)\right), \quad t \geq 0
$$

for a fixed $\lambda>0$. Hence (notice that $h(t) \geq 1$ ),

$$
\begin{aligned}
\exp \left(\frac{\lambda}{q} \int_{0}^{t}[h(s) F(s)]^{q} d s\right) & =\left\{\exp \left(\lambda \int_{0}^{t}[h(s) F(s)]^{q} d s\right)\right\}^{\frac{1}{q}} \\
& =\frac{H_{\lambda}(t)}{h(t)} e^{-(n+1) t} \leq H_{\lambda}(t) e^{-(n+1) t}
\end{aligned}
$$

Here, $q$ is taken such that,

$$
\frac{1}{p}+\frac{1}{q}=1 \text { and } 1<p<\min \left\{\frac{1}{\alpha}, \frac{1}{1-\alpha}, \frac{1}{1-\alpha+\beta}\right\} .
$$

Introduce the complete metric space $\Omega=\left(\chi, d_{\lambda}\right)$, where $\chi$ is the set of all element of $C\left(\mathbb{R}^{\geq 0}, \mathbb{R}\right)$ that behave as $O\left(H_{\lambda}(t)\right)$ when $t$ goes to $+\infty$ and

$$
d_{\lambda}(x, y)=\sup _{t \geq 0}\left\{\frac{|x(t)-y(t)|}{H_{\lambda}(t)}\right\}
$$

for any $x, y \in X$. Since $H_{\lambda}(t) \geq 1$, all the constant functions belong to $X$.

Given the operator $T: \Omega \rightarrow C\left(\mathbb{R}^{\geq 0}, \mathbb{R}\right)$ with the formula

$$
\begin{aligned}
(T x)(t) & =x(0)+\gamma \sum_{k=0}^{n} \mu(k, n, \alpha) t^{n-k} I^{\alpha-\beta+k}[x(t)-x(0)]+I^{\alpha} f(t, x(t)) \\
& =x(0)+\gamma \sum_{k=0}^{n} \mu(k, n, \alpha) \int_{0}^{t} \frac{(t-s)^{\alpha-\beta+k-1}[x(s)-x(0)]}{\Gamma(\alpha-\beta+k)} d s \\
& +\frac{1}{\Gamma(\alpha)} \int_{0}^{t}(t-s)^{\alpha-1} f(s, x(s)) d s .
\end{aligned}
$$


Set,

$$
\begin{aligned}
M & =\max \left\{\frac{|\gamma \mu(k, n, \alpha)|}{\Gamma(\alpha-\beta+k)}: k=0,1, \ldots, n\right\}, \\
& =\max \left\{\frac{\mid \gamma\left(\begin{array}{l}
-\alpha \\
k
\end{array}\right) n !}{(n-k) ! \Gamma(\alpha-\beta+k)}: k=0,1, \ldots, n\right\},
\end{aligned}
$$

then we have the following estimates (notice that $t^{n-k} \leq e^{n t}$ for each $k=0,1,2, \ldots, n$ and $t \geq 0$ )

$$
\begin{aligned}
|(T x)(t)-(T y)(t)| & \leq M e^{n t} \sum_{k=0}^{n} \int_{0}^{t}(t-s)^{\alpha-\beta+k-1}|x(s)-\gamma(s)| d s \\
& +\frac{1}{\Gamma(\alpha)} \int_{0}^{t}(t-s)^{\alpha-1} F(s)|x(s)-\gamma(s)| d s \\
& \leq M e^{n t} \sum_{k=0}^{n} \int_{0}^{t} e^{s}(t-s)^{\alpha-\beta+k-1} F(s) \frac{|x(s)-\gamma(s)|}{e^{s}} d s \\
& +\frac{1}{\Gamma(\alpha)} \int_{0}^{t} e^{s}(t-s)^{\alpha-1} F(s) \frac{|x(s)-\gamma(s)|}{e^{s}} d s \\
& \leq M e^{n t} \sum_{k=0}^{n} I_{k}(t) J(x, y)(t)+I(t) J(x, y)(t),
\end{aligned}
$$

where for each $k=0,1,2, \ldots, n$,

$$
\begin{aligned}
I_{k}(t) & =\left\{\int_{0}^{t}(t-s)^{p(\alpha-\beta+k-1)} e^{p s} d s\right\}^{\frac{1}{p}} \\
& =e^{t}\left\{\int_{0}^{p t}\left(\frac{s}{p}\right)^{p(\alpha-\beta+k-1)} e^{-s} \frac{d s}{p}\right\}^{\frac{1}{p}} \\
& =p^{\frac{1}{q}+\beta-\alpha-k} e^{t}\left\{\int_{0}^{p t} s^{p(\alpha-\beta+k-1)} e^{-s} d s\right\}^{\frac{1}{p}} \\
& \leq p^{\frac{1}{q}+\beta-\alpha-k} e^{t} \Gamma(1-p(1-\alpha+\beta-k))=C(p, \alpha, \beta, k) e^{t},
\end{aligned}
$$

where $C(p, \alpha, \beta, k)=p^{\frac{1}{q}+\beta-\alpha-k} \Gamma(1-p(1-\alpha+\beta-k))$.

$$
\begin{aligned}
J(x, y)(t) & =\left\{\int_{0}^{t}(F(s))^{q} \frac{|x(s)-\gamma(s)|^{q}}{e^{s q}} d s\right\}^{\frac{1}{q}} \\
& =\left\{\int_{0}^{t} \frac{d}{d s}\left(\frac{\exp \left(\lambda \int_{0}^{s}[h(\rho) F(\rho)]^{q} d \rho\right)}{\lambda}\right)\left(\frac{|x(s)-\gamma(s)|}{H_{\lambda}(s)}\right)^{q} d s\right\}^{\frac{1}{q}} \\
& \leq\left\{\int_{0}^{t} \frac{d}{d s}\left(\frac{\exp \left(\lambda \int_{0}^{s}[h(\rho) F(\rho)]^{q} d \rho\right)}{\lambda}\right) d s\right\}^{\frac{1}{q}} d_{\lambda}(x, y) \\
& =\lambda \frac{-1}{q}\left\{\exp \left(\lambda \int_{0}^{s}[h(\rho) F(\rho)]^{q} d \rho\right)\right\}^{\frac{1}{q}} d_{\lambda}(x, y) .
\end{aligned}
$$


Substituting Equation (7) into Equation (10) yields

$$
\begin{aligned}
& J(x, y)(t)=\lambda^{\frac{-1}{q}} e^{-(n+1) t} H_{\lambda}(t) d_{\lambda}(x, y) \\
& \leq \lambda^{\frac{-1}{q}} e^{-t} H_{\lambda}(t) d_{\lambda}(x, y), \quad t \geq 0 . \\
& I(t)=\frac{1}{\Gamma(\alpha)}\left\{\int_{0}^{t}(t-s)^{p(\alpha-1} e^{p s} d s\right\}^{\frac{1}{p}}=\frac{e^{t}}{\Gamma(\alpha)}\left\{\int_{0}^{p t}\left(\frac{s}{p}\right)^{p(\alpha-1)}\left(e^{-s}\right) \frac{d s}{p}\right\}^{\frac{1}{p}} \\
& =\frac{e^{t}}{\Gamma(\alpha)} p^{\frac{1}{q}-\alpha} \Gamma(1-p(1-\alpha))=C_{2}(\alpha, p) e^{t}
\end{aligned}
$$

where $C_{2}(\alpha, p)=\frac{1}{\Gamma(\alpha)} p^{\frac{1}{q}-\alpha} \Gamma(1-p(1-\alpha))$.

Hence, substituting (9), (11), and (12) into inequality (8) leads to

$$
\begin{aligned}
|(T x)(t)-(T y)(t)| & \leq\left(M \sum_{k=0}^{n} C_{1}(p, \alpha, \beta, k)\right) \lambda^{\frac{-1}{q}} H_{\lambda}(t) d_{\lambda}(x, y) \\
& +C_{2}(\alpha, p) \lambda^{\frac{-1}{q}} H_{\lambda}(t) d_{\lambda}(x, y) .
\end{aligned}
$$

Therefore,

$$
d_{\lambda}(T x, T y) \leq\left\{M \sum_{k=0}^{n} C_{1}(p, \alpha, \beta, k)+C_{2}(\alpha, p)\right\} \lambda^{\frac{-1}{q}} d_{\lambda}(x, y)
$$

The formula is valid only if we establish that $T x \in \chi$ whenever $x \in \chi$. This follows from the next estimates

$$
\begin{aligned}
& \left.|(T x)(t)| \leq \mid(T x)(t)-\left(T x_{0}\right)\right)(t)|+|\left(T x_{0}\right)(t) \mid \\
& \leq\left(M \sum_{k=0}^{n} C_{1}(p, \alpha, \beta, k)+C_{2}(\alpha, p)\right) \lambda^{\frac{-1}{q}} H_{\lambda}(t) d_{\lambda}\left(x, x_{0}\right)+h(t) \\
& \leq\left\{\left(M \sum_{k=0}^{n} C_{1}(p, \alpha, \beta, k)+C_{2}(\alpha, p)\right) \lambda^{\frac{-1}{q}} d_{\lambda}\left(x, x_{0}\right)+1\right\} H_{\lambda}(t) \\
& =O\left(H_{\lambda}(t)\right) \text { as } t \rightarrow+\infty .
\end{aligned}
$$

In conclusion, the operator $T: \Omega \rightarrow \Omega$ is a contraction for every

$$
\lambda>\left(M \sum_{k=0}^{n} C_{1}(p, \alpha, \beta, k)+C_{2}(\alpha, p)\right)^{q} .
$$

Its unique fixed point is the unique solution of Equation (2) in $C([0, \infty), \mathbb{R})$.

Example 3.3 The following fractional differential equation:

$$
\left\{\begin{array}{l}
\left(D^{\frac{2}{3}}-t^{3} D^{\frac{1}{2}}\right)[x(t)-x(0)]=\sin \left(\frac{\left(2+t^{2}\right) x}{1+t^{2}}\right), \quad t>0 \\
x(0)=x_{0},\left.I^{\frac{1}{3}} x(t)\right|_{t=0}=0 \text { and }\left.I^{\frac{1}{2}} x(t)\right|_{t=0}=0
\end{array}\right.
$$


satisfying in Theorem 3.2. In this example we have,

$$
f(t, x(t))=\sin \left(\frac{2 x}{1+t^{2}}\right) \text { and hence }|f(t, x)-f(t, y)| \leq \frac{2+t^{2}}{1+t^{2}}|x-y|,
$$

so that $F(t)=\frac{2+t^{2}}{1+t^{2}} \geq 1$ for each $t \in[0,+\infty)$.

\section{Conclusions}

The existence and uniqueness of solutions for the nonlinear fractional differential equations with initial conditions comprising standard Riemann-Liouville derivatives have been discussed in $C([0,+\infty), \mathbb{R})$. In order to obtain the results in this article the contraction principle had important role. Although the present study provides some insights in the equations encountered in the non-global existence solutions, this existence and uniqueness theorem may be explored for other classes of fractional differential equations which encounter in the global existence solutions, like recent contributions $[16,24]$, that is a subject for future study.

\section{Acknowledgements}

The author would like to express his appreciation for the valuable comments of the reviewers which improved the exposition of the article.

\section{Competing interests}

The author declares that they have no competing interests.

Received: 8 December 2011 Accepted: 5 April 2012 Published: 5 April 2012

\section{References}

1. Glockle, WG, Nonnenmacher, TF: A fractional calculus approach of self-similar protein dynamics. Biophys J. 68, 46-53 (1995). doi:10.1016/S0006-3495(95)80157-8

2. Hilfer, R: Applications of Fractional Calculus in Physics. World Scientific, Singapore (2000)

3. Metzler, F, Schick, W, Kilian, HG, Nonnenmacher, TF: Relaxation in filled polymers: A fractional calculus approach. J Chem Phys. 103, 7180-7186 (1995). doi:10.1063/1.470346

4. Podlubny, I: Fractional Differential Equations. Academic Press, San Diego (1999)

5. Poudlubny, I: Geometric and physical interpretation of fractional integration and fractional differentiation. Fract Calc Appl Anal. 5, 367-386 (2002)

6. Kilbas, AA, Srivastava, HM, Trujillo, JJ: Theory and applications of fractional differential equations. In North-Holland Mathematics Studies, vol. 204,Elsevier Science B. V, Amesterdam (2009)

7. Lashmikantham, V, Leela, S, Vasundhara, J: Theory of fractional dynamic systems. Cambridge Academic Publishers, Cambridge (2009)

8. Agarwal, RP, Benchohra, M, Hamani, S: Asurvey on existence result for boundary value problems of nonlinear fractional differential equations and inclusions. Acta Appl Math. 109, 973-1033 (2010). doi:10.1007/s10440-008-9356-6

9. Daftardar-Gejji, V, Babakhani, A: Analysis of a system of fractional differential equations. J Math Anal Appl. 293, 511-522 (2004). doi:10.1016/j.jmaa.2004.01.013

10. Babakhani, A, Enteghami, E: Existence of positive solutions for multiterm fractional differential equations of finite delay with polynomial coefficients. Abstract and Applied Analysis 2009 (2009). Article ID 768920. doi: 10.1155/2009/768920

11. Babakhani, A: Positive solutions for system of nonlinear fractional differential equations in two dimensions with delay. 2010 (2010) Article ID 536317. doi:10.1155/2010/536317

12. Baleanu, D, Golmankhaneh, AK, Nigmatullin, R: Fractional Newtonian mechanics. Central Eur J Phys. 8, 120-125 (2010). doi:10.2478/s11534-009-0085-x

13. Baleanu, D, Trujillo, JJ: A new method of finding the fractional Euler-Lagrange and Hamilton equations within Caputo fractional derivatives. Commun Nonlinear Sci Numer Simul. 15(5), 111-115 (2010)

14. Baleanu, D, Trujillo, JJ: New applications of fractoinal variational principles. Rep Math Phys. 61, 331-335 (2008)

15. Benchohra, M, Hamani, S, Ntouyas, SK: Boundary value problems for differential equations with fractional order. Surv Math Appl. 3, 1-12 (2008)

16. Baleanu, D, Mustafa, OG: On the global existence of solutions to a class of fractional differential equations. Comput Math Appl. 59, 1835-1841 (2010). doi:10.1016/j.camwa.2009.08.028

17. Chang, YK, Nieto, Jj: Some new existence results for fractional differential inclusions with boundary conditions. Math Comput Model. 49, 605-609 (2009). doi:10.1016/j.mcm.2008.03.014

18. Ouahab, A: Some results for fractional boundary value problem of differential inclusions. Nonlinear Anal. 69(11) 3877-3896 (2008). doi:10.1016/j.na.2007.10.021

19. Miller, KB, Ross, B: An introduction to the fractional calculus and fractional differential equations. Wiely, New York (1993)

20. Samko, SG, Kilbas, AA, Marichev, Ol: Fractional integrals and derivatives: Theory and applications. Gordon and Breach, Yverdon (1993) 
21. Zhang, S: Positive solutions to singular boundary value problem for nonlinear fractional differential equation. Comput Math Appl. 59(3), 1300-1309 (2010). doi:10.1016/j.camwa.2009.06.034

22. Feng, $M$, Liu, $X$, Feng, $\mathrm{H}$ : The existence of positive solution to a nonlinear fractional differential equation with integral boundary conditions. Adv Diff Equ 2011, 14 (2011). Article ID 546038, doi:10.1155/2011/546038. doi:10.1186/1687-18472011-14

23. Liu, X, Jia, M: Multiple solutions of nonlocal boundary value problems for fractional differential equations on the halfline. Electron J Qualitative Theory Diff Equ. 56, 1-14 (2011)

24. Feng, $M$, Zhang, $X, G e, W$ : New existence results for higher-order nonlinear fractional differential equation with integral boundary conditions. Boundary Value Problems 2011, 20 (2011). Article ID 720702, doi:10.1155/2011/720702. doi:10.1186/1687-2770-2011-20

25. Belarbi, $A$, Benchohra, $M$, Ouahab, A: Uniqueness results for fractional differential equations with infinite delay in Frechet space. Appl Anal. 85, 1459-1470 (2006). doi:10.1080/00036810601066350

26. Benchohra, M, Henderson, J, Ntouyas, SK, Ouahab, A: Existence esults for fractional order functional differential equations. J Math Anal Appl. 338(2), 1340-1350 (2008). doi:10.1016/j.jmaa.2007.06.021

27. Heymans, N, Podlubny, I: Physical interpretation of initial conditions for fractional differential equations with RiemannLiouville fractional derivative. Rheol, Acta. 45(5), 765-772 (2006). doi:10.1007/s00397-005-0043-5

28. Lashmikantham, V, Vatsala, AS: Basic theory of fractional differential equations. Nonlinear Anal TMA. 69, 2677-2682 (2008). doi:10.1016/.na.2007.08.042

doi:10.1186/1687-1847-2012-41

Cite this article as: Babakhani: Existence and uniqueness of solution for class of fractional order differential equations on an unbounded domain. Advances in Difference Equations 2012 2012:41.

\section{Submit your manuscript to a SpringerOpen ${ }^{\odot}$} journal and benefit from:

- Convenient online submission

- Rigorous peer review

- Immediate publication on acceptance

- Open access: articles freely available online

- High visibility within the field

- Retaining the copyright to your article

Submit your next manuscript at $\boldsymbol{s p r i n g e r o p e n . c o m ~}$ 